access and use health information. Current findings demonstrate scope for HCPs and established healthcare organisations to further utilise YouTube for the dissemination of quality-controlled, evidence-based information.

Disclosure of Interest: None declared

DOI: 10.1136/annrheumdis-2018-eular.7751

\section{SP0132 MEASURING DIGITAL HEALTH LITERACY, WHY AND HOW?}

C. Drossaert. Psychology Health and Technology, University of Twente, Enschede, Netherlands

Digital health literacy or eHealth Literacy refers to a person's ability to search, select, appraise and apply online health information or appropriately use digital health applications. In this presentation I will address the issue of measurement of these skills. First, we will explore why it is important to measure digital health literacy and discuss the different aims of measuring. Second, we will address some of the currently available instruments, including the oldest and most used instrument, the eHealth Literacy Scale or EHEALS Norman \& Skinner, 2006 and some more recent instruments, including the eHealth Literacy Questionnaire, eHLQ Kayser et al. 2018 and the Digital Health Literacy Instrument, DHLI. Van der Vaart \& Drossaert, 2017. Of each instrument, I will briefly discuss its underlying theory, some empirical findings, and its strengths and weaknesses. I will conclude with discussing some general challenges in measuring digital health literacy and directions for future research.

Disclosure of Interest: None declared

DOI: 10.1136/annrheumdis-2018-eular.7798

\section{SP0133 PATIENT EXPERIENCES FROM A TELE-HEALTH INTERVENTION ON DISEASE ACTIVITY IN RA: THE KEEN AND THE RELUCTANT PATIENT}

L.R. Knudsen. Department of Rheumatology, Aarhus University Hospital, Aarhus, Denmark

Background: Recently, the effectiveness of monitoring disease activity in rheumatoid arthritis (RA) through a patient-reported outcome (PRO)-based tele-health follow-up strategy was compared to usual outpatient follow-up in the TeRA trial. Telemedicine interventions require patients taking an active role in the disease course and treatment, and assuming more responsibility for monitoring and identifying signs and symptoms of disease activity. ${ }^{2-3}$ The TeRA study examines the effectiveness of tele-health follow-up, but provides no insight into how patients experience this new approach to disease control.

Objectives: To explore the experiences of a PRO-based tele-health follow-up from the perspective of patients with RA and their experiences of increasing their active role and responsibility for disease control in particular.

Methods: Adopting a strategy of interpretive description, we conducted individual, semi-structured interviews with 15 RA patients participating in the tele-health follow-up. Participants were selected purposively and consecutive from both genders and with various ages, disease durations and disease severity. The analysis was inductive with a constant comparative approach. First, we identified the main themes conveying the participants' experiences. Then, we constructed patient typologies to explain different perspectives on the tele-health follow-up.

Results: Five themes covered the participants' experiences: 'A flexible solution', 'Responsibility','Knowledge of RA', 'Communication and involvement' and 'Continuity'. Two typologies: 'the keen patient' and 'the reluctant patient' represented opposite perspectives and preferences regarding the core value of and approach to the tele-health follow-up.

Conclusions: The participants had positive perceptions of the PRO-based telehealth follow-up and saw it as a flexible and resource-saving solution that can reduce the burden of unnecessary interruptions in everyday life. They reported disadvantages related to missing face-to-face contact with health professionals.

The two typologies, 'the keen' and 'the reluctant' patient, help us understand the patients' different needs, wishes and abilities to take part in tele-health follow-up. Our findings reveal a need for more insight into how tele-health follow-up could be integrated in routine clinical practice, paying special attention to how reluctant patients may be supported.

\section{REFERENCES}

[1] Thurah A, Stengaard-Pedersen K, Axelsen M, Fredberg U, Schougaard LMV, Hjollund NHI, et al. A tele-health follow-up strategy for tight control of disease activity in rheumatoid arthritis: results of the non-inferiority randomised controlled trial (the TeRA study). Arthritis Care Res 2017 [Epub ahead of print]
[2] Wildevuur SE, Simonse LW. Information and communication technologyenabled person-centred care for the "big five" chronic conditions: Scoping review. J Med Internet Res 2015;17:77

[3] Paré G, Mogadem K, Pineau G, St-Hilaire C. Clinical effects of home telemonitoring in the context of diabetes, asthma, heart failure and hyperten sion: A systematic review. J Med Internet Res 2010;12:21.

[4] Thorne S. Interpretive description: Qualitative Research for Applied Practice. New York and London: Routledge; 2016 (2nd ed.).

Disclosure of Interest: None declared DOI: 10.1136/annrheumdis-2018-eular.3066

FRIDAY, 15 JUNE 2018

\section{The rheumatologist-orthopaedic surgeon connexion in secondary fracture prevention}

\section{SP0134 THE CONNEXION BETWEEN FRACTURE CARE AND SECONDARY FRACTURE PREVENTION}

K. Åkesson. Dept Clinical Sciences Malmö, Lund University, Malmo, Sweden

That fracture begets fracture, is today an acknowledged reality. Nevertheless there are still gaps in fracture care and many are far from reaching the optimal treatment pathway. Ultimately, each fracture should be appropriately managed from the moment of the fracture, through the acute management of the patient and the fracture, through to rehabilitation and secondary prevention. Optimisation of every step leads to better post-fracture functioning and quality of life, in addition to a reduced risk of new fracture events where the next fracture often is more severe than the previous.

Fracture treatment has improved with newer implants (plates, screws, nails, joint replacements etc) being developed specifically for fragile bone. Standardising procedures and checklists have made a difference in reducing complications. In the field of anaesthesia advances allows for surgery in the increasingly frail older person with acceptable perioperative risks and outcome. Similarly, tailored medical management is essential also to improve rapid post-operative recovery. The team approach to prompt regaining of function and rehabilitation allows for faster return to the home.

However, it has been more difficult to systematically improve the final step - prevention to avoid recurrence. It is a fact that patients at the highest risk of fracture, those who have already sustained a fracture, have overwhelmingly remain unidentified for osteoporosis treatment and falls prevention. Exceptions exist and they have become examples of best practice; whereby integrated, systematic identification, investigation and intervention were key components for secondary prevention of fractures. The cornerstone in such programs is the fracture coordinator; hence, they are commonly referred to as a fracture liaison service (FLS) They are the link between the orthopaedics and the osteoporosis clinic. Through the development of a best practice framework, the key components for developing secondary fracture prevention programs are outlined and tool kits on how to get started are available. The fundamental component is however, acceptance by the system in order to incorporate prevention as a required part of fracture management.

The presentation will provide an overview of main advances as well as tips on how to move forward.

Disclosure of Interest: K. Åkesson Speakers bureau: Invited lectures for Amgen, Lilly, Radius, UCB DOI: 10.1136/annrheumdis-2018-eular.7834

\section{SP0135 THE EULAR/EFORT RECOMMENDATIONS FOR PATIENTS WITH RECENT FRACTURE}

W.F. Lems, on behalf of Working Group in EULAR EFORt recommendations in patients 50 years and over with a fracture. Rheumatology, location VUmc, Amsterdam Rheumatology and immunolgy Center, Amsterdam, Netherlands

The European League Against Rheumatism (EULAR) and the European Federation of National Associations of Orthopaedics and Traumatology (EFORT) have recognised the importance of optimal acute care for the patient 50 years and over with a recent fragility fracture and the prevention of subsequent fractures in high risk patients, which can be facilitated by close collaboration between orthopaedic surgeons and rheumatologists or other metabolic bone experts. Therefore, the aim was to establish for the first time collaborative recommendations for these patients.

According to the EULAR standard operating procedures for the elaboration and implementation of evidence-based recommendations, 8 rheumatologists from 8 
countries and 10 orthopaedic surgeons from 10 countries met twice under the leadership of 2 conveners, a senior advisor, a clinical epidemiologist and 3 research fellows. After defining the content and procedures of the task force, 10 research questions were formulated, a comprehensive and systematic literature search was performed, and the results were presented to the entire committee. Subsequently, 10 recommendations were formulated based on evidence from the literature and after discussion and consensus building in the group.

The 10 recommendations will be discussed at the meeting; they included appropriate medical and surgical peri-operative care which requires, especially in the elderly, a multidisciplinary approach including orthogeriatric care. A coordinator should build up an organisation with systematic investigations for future fracture risk in all elderly patients with a recent fracture. High-risk patients should have appropriate non-pharmacological and pharmacological treatment to decrease the risk of subsequent fracture.

\section{REFERENCE:}

[1] EULAR/EFORT recommendations for management of patients older than 50 years with a fragility fracture and prevention of subsequent fractures. Ann Rheum Dis. 2017 May;76(5):802-810.

Disclosure of Interest: W. F. Lems Consultant for: Amgen, Eli Lilly, Merck, Speakers bureau: Amgen, Eli Lilly, Merck

DOI: 10.1136/annrheumdis-2018-eular.7721

FRIDAY, 15 JUNE 2018

\section{What's new: Latest advances in treatment in JIA and osteoarthritis}

\section{SP0136 LAST ADVANCES IN TREATMENT AND MANAGEMENT OF OSTEOARTHRITIS}

M. Kloppenburg. Rheumatology, Leiden University Medical Center, Leiden, Netherlands

Osteoarthritis is a highly prevalent disease that results in a considerable disease burden for patients that suffer from this disease. Osteoarthritis can affect any joint, but is especially prevalent in the knee, hips and hands. The management for osteoarthritis includes non-pharmacological, pharmacological and surgical options. But options depend on the location of osteoarthritis, since not all treatment options are equally effective for patients with different osteoarthritis phenotypes. Fortunately, the number of high-quality clinical trials has increased in the last years and have increased our insight in potential effective treatments for osteoarthritis. Non-pharmacological options include information and education, exercise possibly in combination with weight reduction in overweight patients with knee osteoarthritis or assistive technology in patients with hand osteoarthritis. Regarding effective pain alleviating medication research, including systematic reviews, network analyses and randomised clinical trials, has increased our insight in the clinical efficacy of different medications. This has led to the discussion about the role of acetaminophen in osteoarthritis. New pain alleviating medication has been developed and is currently investigated. Furthermore, currently used medication is investigated for alternative ways of application. The ultimate requirement to have a disease modifying drug available is not yet met, but studies have been undertaken and are ongoing to investigate disease modifying potential.

Disclosure of Interest: None declared

DOI: 10.1136/annrheumdis-2018-eular.7780

\section{FRIDAY, 15 JUNE 2018}

\section{Assessment and prevention of RMDs, what have we learned?}

\section{SP0137 PREVENTION OF RMDS - WHAT HAVE WE LEARNED?}

S.M. Verstappen ${ }^{1,2} .{ }^{1}$ NIHR Manchester Biomedical Research Centre, Central Manchester University Hospitals NHS Foundation Trust, Manchester Academic Health Science Centre; ${ }^{2}$ Arthritis Research UK Centre for Epidemiology, Centre for Musculoskeletal Research, Division of Musculoskeletal and Dermatological Sciences, The University of Manchester, Manchester, UK

Rheumatic and Musculoskeletal Diseases (RMDs) cause the greatest burden of disability in Europe and is increasing. The WHO Europe Action Plan for the Prevention and Control of Non-communicable Diseases recently recognised this burden and recommends action to promote prevention and improve health in the general population and those with musculoskeletal health. Targeted screening and prevention in individuals at high-risk of developing RMDs will only be successful if there is a good understanding of the underlying mechanisms of the disease and of possible genetic and environmental risk factors associated with the risk of developing RMDs. The main focus of this presentation is on the evidence of the association between modifiable lifestyle factors and the risk of developing RMDs and the effectiveness of drugs administered during the preclinical phase of RMDs.

Disclosure of Interest: None declared

DOI: 10.1136/annrheumdis-2018-eular.7783

\section{SP0138 WHY NOT ALL EFFECTIVE INTERVENTIONS ARE WORTHWHILE}

F. Guillemin. Université de Lorraine, APEMAC, Nancy, France

Economic decisions in the health sector should be made to reach both efficiency and improvement of health in the most equitable way in the population. Comparative assessment of innovation with usual or current standard practice allows measuring the incremental benefit per individual. The comparison should bear both on effectiveness, ie health effect, and on costs of alternatives, considering appropriate stakeholders and payers. Moreover, according to diseases and treatment target, the time horizon should be relevant to its expected effect, from a few weeks for painkillers to a lifetime for disease modifying drugs in inflammatory arthritis, joint prosthesis in osteoarthritis, or prevention of osteoporosis.

Various scenarios can result from an economic analysis, with four possibilities for a new treatment or health care technology: more efficient at lower cost (optimal), less efficient at higher cost (worse issue), less efficient at lower cost (needing a decision to lose some effectiveness), or more efficient at higher cost - ie costeffective - (needing to identify what the society is ready to pay off). This is done by calculating an incremental cost-effectiveness ratio, illustrating a marginal benefit per individual, at best using data from clinical trials. Therefore, not all effective interventions can be candidate for diffusion.

Because there is a need to make decisions before larger diffusion of the innovation, a set of methods are also available to anticipate its potential benefit, using a modelling approach. Sensitivity analyses are also conducted in varying conditions of application in reasonable range, using deterministic and stochastic methods. More recently, another perspective has been developed, ie the calculation of the net budgetary impact. This approach allows an extension of the costs to the targeted people in the whole population at a country level. It should take all indirect costs into account. Using these approaches, it is possible to observe that sometimes promising health technology sometimes need to be abandoned, given the too high cost to society for low additional benefit to individuals.

Disclosure of Interest: None declared

DOI: 10.1136/annrheumdis-2018-eular.7788

\section{FRIDAY, 15 JUNE 2018}

\section{High-end imaging: looking for the invisible}

\section{SP0139 THE ROLE OF PHAGOCYTES AT THE INFLAMMATORY SITE}

S. Uderhardt, on behalf of Ronald N Germain. Laboratory of Systems Biology, National Institute of Allergy and Infectious Diseases, Bethesda, USA

Inflammation is a highly conserved, multicellular response to infection or injury ensuring host defense and tissue integrity. Immune cell activation, however, can cause substantial collateral damage, often further amplifying the inflammatory response and significantly contributing to disease pathology (e.g. influenza, myocardial infarction). Hence, mechanisms are required not only to promote and resolve inflammation, but also to regulate the primary events that initiate this proc ess in order to avoid unwanted and potentially harmful immune responses.

Using state-of-the-art intravital and static multi-parameter imaging techniques in mice, our lab seeks to understand the complex interactions and functions of different immune cells types in the execution and regulation of the inflammatory responses to sterile damages in peripheral tissues. With primary focus on the innate immune system, we're particularly interested in the dynamic interplay of embryonically-derived tissue macrophages and recruited neutrophils, which can prevent unwanted immune cell activation and thereby fine-tune the threshold for the onset of damaging inflammation.

This presentation will provide novel insights into the multi-layered regulation of the very initial steps in an inflammatory response to tissue damage, and will further discuss the differential roles of different populations of phagocytes at sites of inflammation. 